\title{
ESTUDIO DE LA SENSIBILIDAD DEL OXÍGENO DISUELTO Y LA DEMANDA BIOQUÍMICA DE OXÍGENO EN AGUAS TROPICALES DEL PACÍFICO
}

\author{
Alexander Navarrete Muñoz * \\ División Protección del Medio Marino, Centro Control Contaminación del Pacífico - CCCP \\ Vía El Morro, Capitanía de Puerto, San Andrés de Tumaco, Nariño, Colombia \\ Tél: +57 (2) - 72726 37, Fax: +57 (2) - 7271180 \\ e-mail: cccp@ cccp.org.co \\ anavarrete@ cccp.org.co *
}

Recibido en octubre de 2003; aceptado en diciembre de 2003

\section{RESUMEN}

\begin{abstract}
En este trabajo se expone el procedimiento para evaluar la sensibilidad a nueve variables diferentes del modelo de evolución de oxígeno del paquete AQUALAB. La zona de estudio se ubica en aguas tropicales del Pacífico colombiano. Se presenta el diseño experimental realizado para separar los efectos de cada variable. Adicionalmente, se define un factor de sensibilidad que a partir de un cambio de las variables evalúa la mayor o menor alteración de resultados. Finalmente, se establece cuáles son las variables más sensibles y se discute la influencia de la localización del punto de registro y el estado de marea en la sensibilidad.
\end{abstract}

Palabras claves: Calidad de Aguas, Modelación, Análisis de Sensibilidad, Aguas Tropicales, Aguas Servidas, Diseño Experimental.

\begin{abstract}
In this work the procedure used to evaluate the sensitivity of the AQUALAB package's oxygen evolution model to nine different variables is exposed. The study area is located in tropical waters in the Colombian Pacific Ocean. The experimental design made to separate the effects of every variable is presented. Additionally a sensitivity factor which from a change in the variables evaluates the greater or smaller alteration of the result, is defined. Finally, The more sensitive variables are established, and the point location and tide phase influence is discussed.
\end{abstract}

Key words: Water Quality, Modeling, Sensitivity Analysis, Tropical Waters, Waste Waters, Experimental Design. 


\section{INTRODUCCIÓN}

El auge de los modelos de calidad de aguas se debe a que se han constituido en una herramienta rápida y económica para el estudio del comportamiento de las variables físicas y bioquímicas de los cuerpos de agua.

Sin embargo, para obtener resultados confiables de un modelo es necesario llevar a cabo una calibración de sus componentes hidrodinámicos y cinéticos. Estos componentes basan su estructura en ecuaciones semiempíricas que requieren, a su vez, la determinación de algunos coeficientes para describir de manera apropiada la variable de interés.

Esta determinación se realiza a través de mediciones in situ, acompañadas por un proceso de tanteo y error que se realiza con el modelo a calibrar. Para esto se comparan condiciones medidas en el lugar de estudio con las simuladas por el modelo. Los coeficientes con los que se alcance el mayor ajuste serán los usados en posteriores simulaciones (EPA, 1990).

Para obtener un modelo de calidad de aguas correctamente adaptado a las condiciones del Pacífico colombiano se ha calibrado el modelo de consumo de oxígeno del sistema AQUALAB desarrollado por la Universidad de Cantabria.

El entorno de trabajo AQUALAB contiene diversos modelos numéricos, que permiten la definición de las características hidrodinámicas del medio marino 0 estuarino, así como el transporte y evolución de sustancias contaminantes o de los contenidos de oxígeno disuelto, OD, y demanda bioquímica de oxígeno, DBO.

AQUALAB describe las condiciones hidrodinámicas mediante las ecuaciones de ondas largas, que resuelve el modelo numérico H2D, en las cuales la hipótesis inicial es $L \gg$ h; siendo $L$ la longitud de la onda cuya propagación se estudia y $h$ la profundidad sobre la que se propaga (AQUALAB, 2001).

Los resultados del modelo para una variable de salida determinada (OD o DBO) cambiarán, en mayor o menor proporción, dependiendo de la variación del valor de uno o varios de los coeficientes. Esto, debido a que existen coeficientes a los que un modelo es más sensible (EPA, 1990).

Así, para conocer la influencia de cada coeficiente sobre los resultados del modelo es necesario reali- zar un análisis de sensibilidad, consistente en la investigación del cambio en el valor de los coeficientes del modelo sobre el resultado de la simulación.

Análisis de sensibilidad de modelos de calidad de aguas superficiales se han realizado en diferentes lugares (Malone et al., 2000). Este trabajo se realiza, típicamente, variando los valores de entrada al modelo en algún porcentaje positivo o negativo y estudiando el resultado obtenido luego de la simulación (EPA, 1990).

En este trabajo se presentará la metodología usada para realizar el análisis de sensibilidad del modelo de consumo de oxígeno en la bahía de Tumaco por medio del sistema AQUALAB. Se parte de previa calibración del modelo hidrodinámico H2D, para simular el componente advectivo del modelo en el área de estudio (Málikov, 2002). Además, se presentan y discuten los resultados obtenidos después de aplicar dicha metodología.

\section{ÁREA DE ESTUDIO}

Ya que se desea contar con un modelo de consumo de oxígeno adaptado a las condiciones propias del Pacífico colombiano se escogió un lugar que, además de ser representativo, estuviese lo suficientemente documentado como para llevar a cabo el trabajo de calibración. El municipio de Tumaco ha sido estudiado de tal manera que se conoce el comportamiento de variables tales como el OD, la $\mathrm{DBO}$, la temperatura, etcétera. Lo que le convierte en el sitio idóneo para llevar a cabo esta labor en el Pacífico colombiano.

La mayor parte de la población de Tumaco se asienta en las islas de Tumaco y El Morro, por esta razón se registra la información de las simulaciones en tres puntos de las mismas (Fig. 1).

La población y su actividad producen vertimientos de aguas servidas que, en su mayor parte, son arrojadas al mar sin tratamiento previo. Estos vertidos se representan en los puntos señalados en la Figura1.

Gutiérrez y Ríomalo reportaron materia orgánica en la bahía de Tumaco, proveniente de la comercialización de productos hidrobiológicos, principalmente. Estos datos, junto con estimaciones de descargas de aguas residuales domésticas se presentan en la Tabla 1, que especifica el caudal correspondiente a los vertidos y la concentración de DBO asociada. 
Es interesante notar en la Tabla 1 que el punto de vertido 1 presenta una alta concentración de DBO, pero un caudal bajo. Este punto corresponde a una empresa que produce harina de pescado (Proteimar) que opera entre siete y diez días al mes. Lo anterior se refleja en que, a pesar de las altas cargas contaminantes, se tenga relativamente poco caudal de vertimiento (Gutierrez y Ríomalo, 2000).

\section{METODOLOGÍA}

\section{Ecuaciones base}

El análisis de sensibilidad se realiza para el modelo de evolución del OD del sistema AQUALAB. Éste arroja como resultado el cambio de la concentración de OD y DBO en la malla de cálculo. AQUALAB se fundamenta en las siguientes ecuaciones (Revilla etal, 2001):

$$
\begin{gathered}
\frac{d(O D)}{d t}=K_{l}\left(C_{s}-O D\right)-D B O C+F-R-D O S \\
\frac{d(D B O C)}{d t}=-K_{2}(D B O C) \\
K_{l}=13 \cdot \frac{U^{0.5}}{H^{1.5}}+\frac{W}{H}
\end{gathered}
$$

$C_{S}=14,652-0,0841 S+T[0,0026 S-0,41022+$

$+T(0,007991-0,0000374 . S-0,000077774 T)]$

$$
K_{2}=K D B O\left(\theta^{T}\right)
$$

Donde:

$$
\begin{aligned}
& K_{1} \quad \text { es el coeficiente de reaireación, } \\
& \text { [día }{ }^{-1} \text { ] } \\
& C_{s} \quad \text { es la concentración de OD a } \\
& \text { saturación, [mg/L] } \\
& O D \quad \text { es la concentración de OD, [mg/L] } \\
& \triangle B O C \text { es la demanda bioquímica de } \\
& \text { oxígeno, [mg/L] } \\
& F \quad \text { es la producción de oxígeno por } \\
& F=0 \quad \rightarrow \alpha<0 \\
& F=F_{\text {máx }} \operatorname{sen}(\alpha) \rightarrow \alpha>0 \\
& F_{\text {máx }} \quad \text { es la máxima producción de } \\
& \text { oxígeno por fotosíntesis a medio- } \\
& \text { día, }\left[\mathrm{g}_{2} / \mathrm{m}^{2}\right] \\
& \alpha \quad \text { es la inclinación solar respecto al } \\
& \text { horizonte. } \\
& R \quad \text { es el consumo de oxígeno por } \\
& \text { respiración, }\left[\mathrm{gO}_{2} / \mathrm{m}^{2}\right] \\
& \text { DOS es la demanda de oxígeno por } \\
& \text { fondos, }\left[\mathrm{gO}_{2} / \mathrm{m}^{2}\right. \text { día] }
\end{aligned}
$$

es la constante de oxidación de la materia orgánica, [día ${ }^{-1}$ ]

KDBO constante base de oxidación de la materia orgánica

$\theta^{T} \quad$ factor de corrección por temperatura para DBO ?

U

es la profundidad, [m]

es la velocidad media del viento, $[\mathrm{m} / \mathrm{s}$ ]

S es la salinidad del agua, $\left[\%_{0}\right]$

$\mathrm{T}$ es la temperatura del agua, $\left[{ }^{\circ} \mathrm{C}\right]$

La dependencia de la temperatura es contabilizada, en el modelo, en los procesos de consumo de oxígeno por respiración y de oxidación de materia orgánica. Esto se logra multiplicando éstos factores por sendas constantes de corrección. Dichas constantes son también incluidas en el análisis de sensibilidad.

\section{Diseño experimental}

Con el objeto de evaluar el grado de influencia que cada variable de estudio (nueve en total) tiene sobre los resultados del modelo de evolución de oxígeno, se debe ejecutar el modelo probando cada variable en uno de los valores escogidos para el estudio.

Se decidió usar tres valores para cada variable, de tal manera que se cubriera un rango de acuerdo a valores obtenidos en campañas de medición o recomendados por literatura especializada (EPA, 1985). De estos tres, uno fue el valor mínimo del rango, otro el máximo y uno adicional que se consideraría como base para las simulaciones.

Para la selección de los valores de cada variable se cubrió el rango teórico o experimental planteados en literatura relacionada con el tema. Los documentos en los que se fundamentó la selección se referencian en la última columna de la Tabla 2.

Sin embargo, si dos variables o más se cambian al mismo tiempo será imposible separar sus efectos matemáticamente (Math works, 1999). Por lo tanto se planeó una serie de ejecuciones del modelo en las que se alimentara el modelo con uno de los tres valores establecidos para cada una de las variables (Tabla 2).

Las variables restantes conservaron su valor base de acuerdo a valores obtenidos en campañas de medición o recomendados por literatura especiali- 
zada (EPA, 1985). Esto resultó en 27 tratamientos (ejecuciones), cuyos resultados serían la base del análisis (Tabla 2).

\section{Evaluación de los resultados}

El grado de sensibilidad se evaluó tomando como base la magnitud del cambio del resultado, a un cambio determinado en los valores de entrada de una cierta variable. Partiendo del método sugerido por Perera y NG (2001), se definió el siguiente factor de sensibilidad:

$$
F S=\frac{\text { Desv Estandar }(\text { valores_salida })}{\text { Desv Estandar }(\text { valores_entrada })}
$$

Así, al terminar las 27 ejecuciones del modelo, se tomaron los tres valores de entrada para una determinada variable y se calculó la desviación estándar. De igual manera se procedió para los resultados correspondientes. Dado que el modelo simula la dinámica del oxígeno a medida que cambia la marea, los resultados se discriminaron en marea baja y alta.

Posteriormente, se analizaron los resultados, basándose para ello en el valor del factor de sensibilidad (FS). A mayor valor que tuviera el factor para una variable, en comparación a las otras, mayor resultaría la sensibilidad del modelo a aquella.

\section{RESULTADOS Y DISCUSIÓN}

Los resultados del análisis de sensibilidad se presentan en las gráficas de la Figura 2. Debe tenerse en cuenta que se tomaron tres puntos de registro por lo tanto cada uno presenta resultados para marea alta y baja.

Puede observarse que las variables a las que el $O D$ y la DBO fueron más sensibles en las ejecuciones son Kresp, KDBO y KDBO Temp Sin embargo, aunque el oxígeno es sensible a KDBO y KDBO Temp, la DBO no lo es para el caso de Kresp. Esto muestra que a pesar de la gran sensibilidad del sistema a Kresp, la DBO es prácticamente insensible a un cambio en ella.

La independencia de la DBO observada en la Figura 2.C resulta evidente si se tiene en cuenta que su comportamiento se describe por la ecuación 5 del modelo, que incluye, además de la constante de oxidación, una corrección por temperatura. Así, una manera de alterar la concentración de DBO en mayor medida, sería cambiar la velocidad y/o concentración de las descargas de aguas residuales.

Los puntos de registro al tener diferentes localizaciones presentan sensibilidades algo diferentes. Así, para el caso del OD el más sensible a la variable Kresp, en marea alta, fue el punto ubicado en la playa de La viciosa $(F S=3.078)$; mientras que el de menor sensibilidad relativa fue el de la playa de El Morro ( $F S=2.489$ ). De igual forma sucede con $\mathrm{KDBO}_{\text {Temp }}$ en marea alta.

Esto, en principio, parece asociarse a que la mayor parte de los vertimientos se ubicaron en la isla de Tumaco, así los sitios como La Viciosa presentan mayor sensibilidad al compararlos con otros donde existen menor cantidad de vertidos como la playa de El Morro.

Adicionalmente, las gráficas muestran que los mayores cambios de las simulaciones de DBO y OD se presentan para los resultados de marea alta.

Lo anterior permite definir como las variables de calibración más importantes: Kresp, KDBO y KDBO Temp. Por su parte, parámetros como la temperatura, la salinidad, la velocidad del viento y la velocidad de fotosíntesis, pueden permanecer en su valor base durante el transcurso de la calibración.

\section{CONCLUSIONES}

- Al aplicar el modelo de evolución de oxígeno de AQUALAB a aguas tropicales, las variables a las que éste es más sensible son: constante de corrección de respiración por temperatura (Kresp), velocidad de oxidación de DBO (KDBO) y la constante de corrección de DBO por temperatura (KDBO Temp).

- La sensibilidad de la DBO y el OD cambia con la posición y el estado de la marea.

- La calibración debe dedicar un mayor esfuerzo en calibrar las constantes Kresp, KDBO y KDBO Temp.

- El estudio de la sensibilidad de un modelo, por sencillo que sea, con respecto a las diferentes variables de entrada en una zona costera en la que luego se prevea su aplicación, es valioso debido a que se puede concentrar el esfuerzo de medición de datos sobre aquellas variables que más influencia pueden tener en los resultados fi- 
nales. En este caso se observa una fuerte dependencia del factor corrector por temperatura de la tasa de respiración, con respecto al resto de las variables contempladas.

\section{REFERENCIAS BIBLIOGRÁFICAS}

AQUALAB. 2001. Manual de referencia. Departamento de Ciencias y Técnicas del Agua y del Medio Ambiente. Universidad de Cantabria, Santander. España.

Centro Control Contaminación del Pacífico. 2000. Modelo Calidad de Aguas para la Ensenada de Tumaco y Plan de Seguimiento y Vigilancia de la Contaminación Marina en el Pacífico Colombiano. Informe Técnico. San Andrés de Tumaco: CCCP.

Environmental Protection Agency. 1985. Rates, constants, and kinetics formulations in surface water quality modeling.

Environmental Protection Agency. 1990. Technical guidance manual for performing waste load allocations. Book III-Part 2.

Eslava, J. 1994. Climatología del Pacífico Colombiano. Academia Colombiana de Ciencias Geofísicas. Colección Eratóstenes No 1. Bogotá DC. Colombia.
Gutiérrez, M y A. Ríomalo. 2000. Diagnóstico Ambiental San Andrés de Tumaco. Corponariño. Tumaco, Nariño. Colombia. 155 pp.

Málikov, I. 2002. Resultados de la Comparación de la Aplicabilidad de los Modelos Desarrollados para la Bahía de Tumaco. Informe Técnico. CCP: San Andrés de Tumaco.

Malone, R., L. Ma, L. Ahuja and K. Rojas. 2000. Evaluation of the Root Zone Water Quality Model (RZWQM): A Review. ASAE Paper No. 99-2145.

Math works. 1999. Matlab, Statistics toolbox: user's guide.

Perera, B. and A. Ng. 2001. River Water Quality Modelling - Parameter Uncertainty, Sensitivity and Estimation. Water Pollution 2001, Sixth International Conference on Modelling, Measuring and Prediction of Water Pollution, Rhodes, Greece, 17-19 Sep 2001, pp.187-196.

Revilla, J., R. Medina, K. Koev, A. Roldán, J. Juanes, C. Álvarez. 2001. Metodología de Estudio de los Saneamientos Litorales. Universidad de Cantabria, Santander. España. 200 pp. 
FIGURAS Y TABLAS

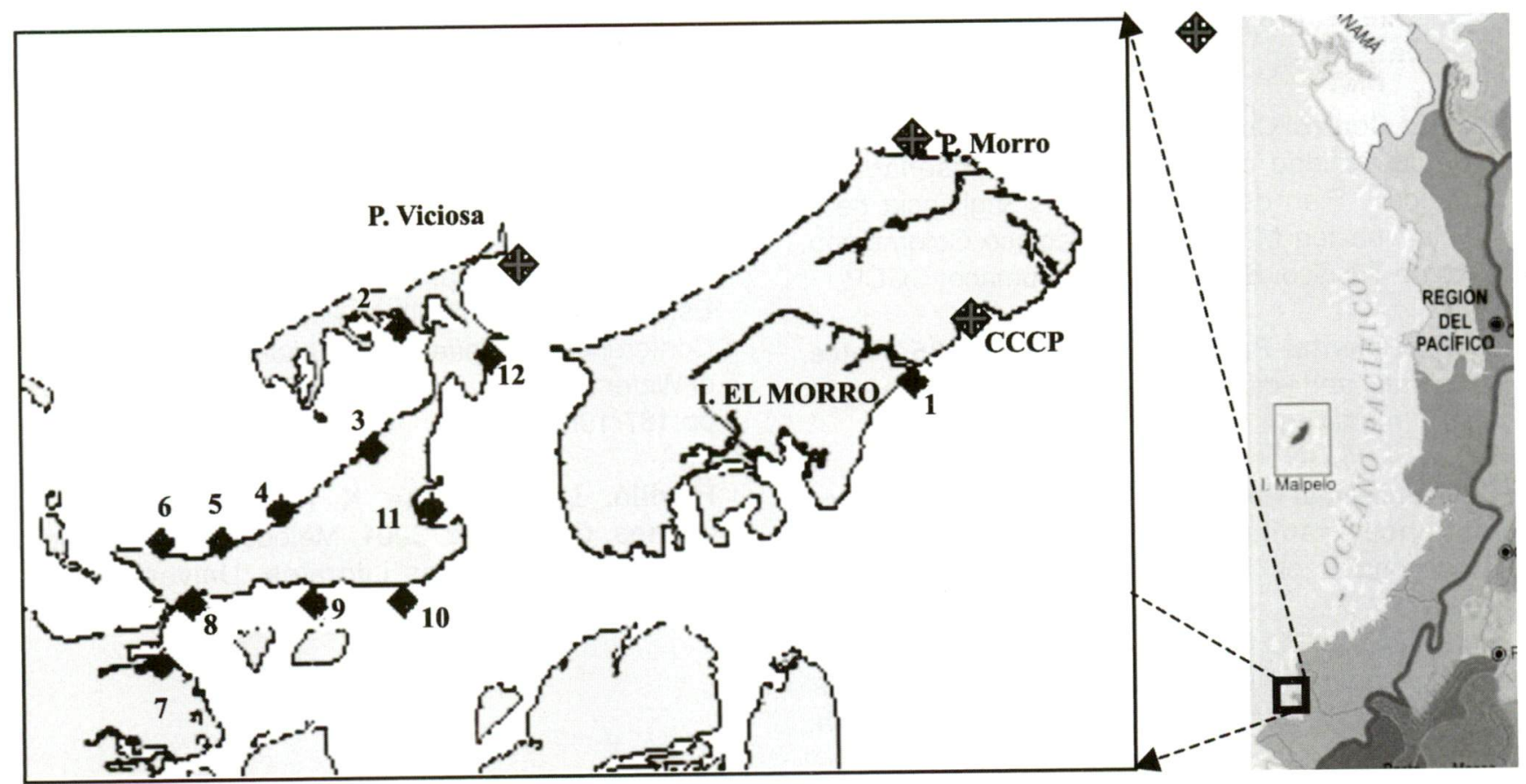

Figura 1. Ubicación del área de estudio y de los puntos de registro ( ) y vertido ( ) para el análisis de sensibilidad. 

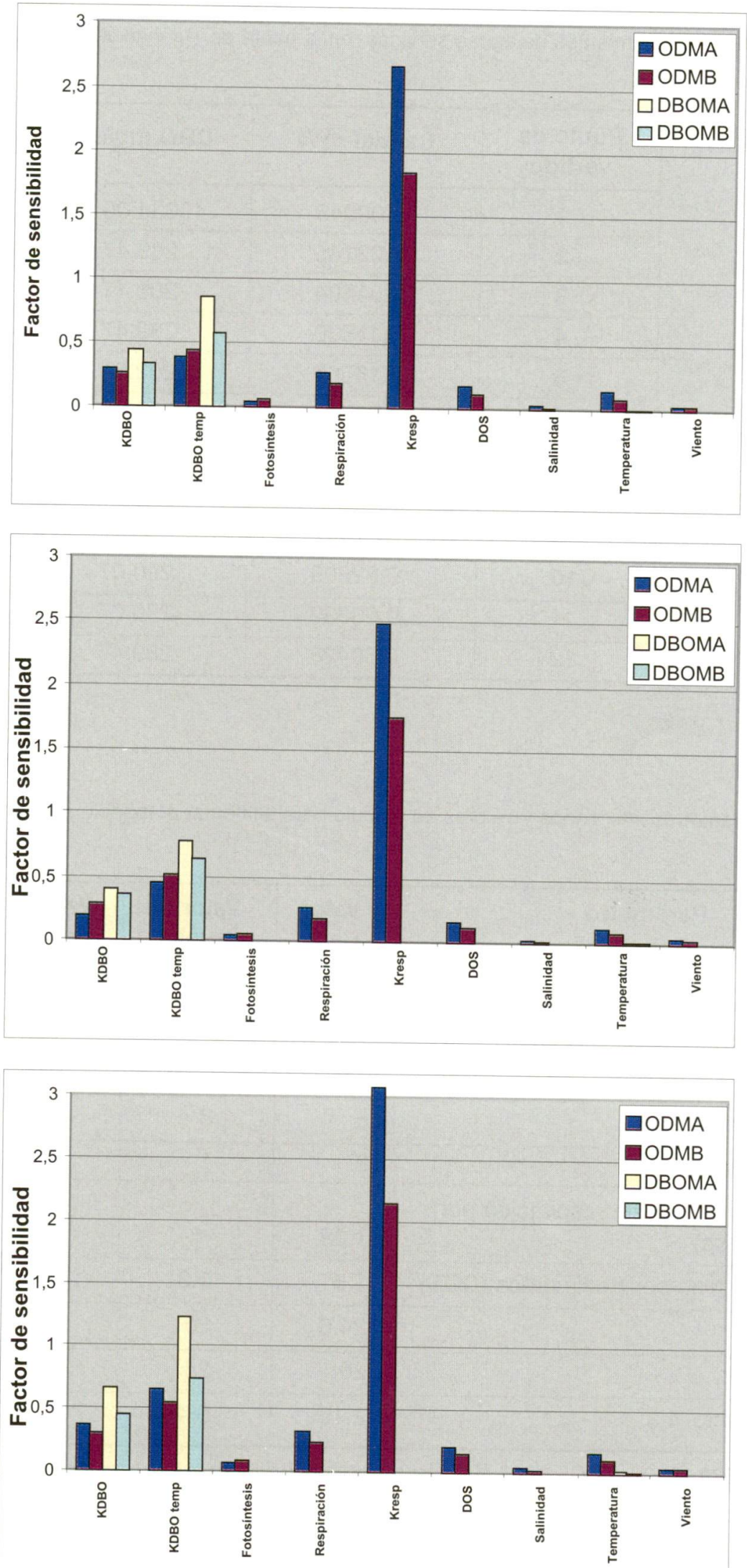

Figura 2. Análisis de sensibilidad. A) Muelle del CCCP; B) Playa de El Morro, y C) Playa de La Viciosa. ODMA: oxígeno disuelto en marea alta; ODMB: oxígeno disuelto en marea baja; DBOMA: DBO en marea alta, y DBOMB: DBO en marea baja. 
Tabla 1. Puntos de vertimientos de aguas servidas, en la bahía de Tumaco, alimentados al modelo.

\begin{tabular}{|c|c|r|}
\hline $\begin{array}{c}\text { Punto de } \\
\text { vertido }\end{array}$ & Caudal $\mathbf{m}^{3} / \mathbf{s}$ & DBO $\mathbf{~ m g} / \mathbf{L}$ \\
\hline $\mathbf{1}$ & 0.00049 & 40034.00 \\
\hline $\mathbf{2}$ & 0.02145 & 289.47 \\
\hline $\mathbf{3}$ & 0.01404 & 289.47 \\
\hline $\mathbf{4}$ & 0.11530 & 289.47 \\
\hline $\mathbf{5}$ & 0.16565 & 202.37 \\
\hline $\mathbf{6}$ & 0.06416 & 289.47 \\
\hline $\mathbf{7}$ & 0.10181 & 289.47 \\
\hline $\mathbf{8}$ & 0.20256 & 289.47 \\
\hline $\mathbf{9}$ & 0.27412 & 289.47 \\
\hline $\mathbf{1 0}$ & 0.02609 & 290.07 \\
\hline $\mathbf{1 1}$ & 0.03339 & 290.07 \\
\hline $\mathbf{1 2}$ & 0.50426 & 289.47 \\
\hline
\end{tabular}

Tabla 2. Parámetros analizados y valores de estudio para alimentar el modelo de calidad de aguas.

\begin{tabular}{|l|l|c|c|c|}
\hline \multicolumn{1}{|c|}{ Parámetro } & $\begin{array}{c}\text { Valor } \\
\text { Base }\end{array}$ & $\begin{array}{l}\text { Valor de } \\
\text { Prueba 1 }\end{array}$ & $\begin{array}{l}\text { Valor de } \\
\text { Prueba 2 }\end{array}$ & Referencia \\
\hline Velocidad de oxidación de DBO (KDBO) & 0.15 & 1 & 1.85 & $* * *+$ \\
\hline $\begin{array}{l}\text { Constante de corrección de DBO por } \\
\text { temperatura (KDBO Temp.) }\end{array}$ & 1.07 & 0.99 & 1.15 & $* * *$ \\
\hline Velocidad de Fotosíntesis & 6 & 3 & 10 & $* \%$ \\
\hline Velocidad de Respiración & 2 & 1 & 7 & $* \%$ \\
\hline $\begin{array}{l}\text { Constante de corrección de respiración por } \\
\text { temperatura (Kresp) }\end{array}$ & 1.08 & 1 & 1.1 & $*$ \\
\hline Demanda de oxígeno por Sedimentos (DOS) & 1.5 & 0.01 & 10 & $* * * \mathrm{~m}$ \\
\hline Salinidad & 24.6 & 20 & 35 & + \\
\hline Temperatura & 26 & 24 & 28 & + \\
\hline Velocidad del viento 4.3 & 2.15 & 6.45 & ++ & \\
\hline
\end{tabular}

*EPA, 1985; ** EPA 1990; +CCCP 2002; ++Eslava $1994 \%$ Revilla et al 2001 mediciones 